\title{
PLEUROSCOPY IN EXUDATIVE PLEURAL EFFUSION: A NORTHERN MALAYSIA EXPERIENCE
}

\author{
A Alaga ${ }^{1 *}$, KA Hamzah $^{1}$, EL Tan $^{1}$, MA Ibrahim ${ }^{1}$, MRN Md Nazri ${ }^{1}$ \\ ${ }^{1}$ Respiratory Department, Hospital Sultanah Bahiyah, Alor Setar, Kedah
}

\begin{abstract}
*Corresponding author:
Dr. Arvindran Alaga, Respiratory Department, Hospital Sultanah Bahiyah, Alor Setar, Kedah,

Malaysia. Email: Arvindran_82@yahoo.com
\end{abstract}

DOI: https://doi.org/10.32896/tij.v1n2.1-6

Published: 31.12 .2021

\begin{abstract}
Background: Pleuroscopy is an investigation modality available for further evaluation of exudative pleural effusion. The aim of this study is to determine the diagnostic yield and the common cause of pleural effusion in patients who underwent pleuroscopy in the Respiratory department of Hospital Sultanah Bahiyah.

Methods: This is a retrospective descriptive analysis study of 105 patients who underwent pleuroscopy in Hospital Sultanah Bahiyah between July 2014 and Dec 2016. Hospital Sultanah Bahiyah is the sole centre performing this procedure in Northern Malaysia.

Result: Biopsies were carried out in $92 \%$ of the cases with a diagnostic yield of up to $90 \%$. The commonest finding was malignancy (53\%) with adenocarcinoma being the commonest subtype followed by granulomatous inflammation (18.6\%) which is expected in Malaysia given our burden of tuberculosis. Majority of our patients were male (57.1\%) with a mean age of 60 years old. With regards to safety profile, re-expansion pulmonary oedema with no procedure related mortality had occurred in one patient as a complication

Conclusion: Pleuroscopy is a safe procedure. Despite the high tuberculosis burden in Malaysia, the commonest cause of exudative pleural effusion in this study is malignancy. Therefore, pleuroscopy should be considered in all cases of exudative pleural effusion.
\end{abstract}

Keywords: Exudative pleural effusion; Pleuroscopy, Lung Malignancy, Tuberculosis

\section{BACKGROUND}

Exudative pleural effusions are a common encounter in daily practice. The etiology varies across different parts of the world. While pneumonia, malignancy, and thromboembolism account for most exudative effusions in the United States [4], tuberculosis (TB) is the most frequently encountered etiology in other developed and developing countries [5]. In general, the first line of investigation in most pleural effusions are diagnostic thoracocentesis. However, in some cases, especially in cases of exudative pleural effusion this procedure alone is inadequate in determining the etiology of the pleural effusion, hence requiring further investigation such as pleural biopsy. This can be done either through a blind biopsy using Abram's needle or via pleuroscopy.

Pleuroscopy or medical thoracoscopy is a form of a minimally invasive procedure that allow direct visualisation and biopsy of the pleura. The first pleuroscopy was introduced by HansChristian JacÖbaeus in 1910 for the replacement of pleural fluid with air as a treatment of tuberculous pleural effusion [1, 2]. Medical thoracoscopy can be performed under local anesthesia and conscious sedation by experienced pulmonologists. 
In Malaysia, pleuroscopy was first introduced in 2004 and were only available in certain hospitals. The service was introduced in Hospital Sultanah Bahiyah in 2014.The objective of this study is to determine the yield and safety of medical pleuroscopy as a diagnostic tool for exudative pleural effusion and to determine the common etiologies for exudative pleural effusion amongst our patient population. To our knowledge, this is the first study from Northern Malaysia and Peninsula Malaysia to analyze the diagnostic yield and safety profiles of this procedure in our local population.

\section{METHODS AND DATA COLLECTION}

This is a retrospective descriptive study of all patients that underwent pleuroscopy. This data is obtained from the respiratory department Hospital Sultanah Bahiyah, Alor Setar (HSB) as part of the investigation for exudative pleural effusion. The diagnosis of exudative pleural effusion was based on Light's criteria.

The study was conducted at Respiratory Department Hospital Sultanah Bahiyah, which is the only center performing pleuroscopy in the state of Kedah, and it caters for patients from the state of Kedah and Perlis, the most northern part of Malaysia peninsula.

All pleuroscopy records in Hospital Sultanah Bahiyah (HSB) from July 2014 until Dec 2016 were retrieved from the bronchoscopy database and patients' names, identification card number and registration number were recorded. Patient data was used to trace the result of pleural fluid analysis and biopsies taken during pleuroscopy. Additional informations were obtained from discharge and follow up notes.

We analysed our data using SPSS version 20. Ccontinuous data were presented in the form of mean and SD while categorical data were presented in number and percentage. The ccomparison between 2 groups were made using unpaired t test for continuous data and chi squared test for categorical data. The significant level is set at 0.05

\section{THORACOSCOPIC PROCEDURE}

Medical pleuroscopy is performed in the bronchoscopy suite by respiratory physician under local anaesthesia in this hospital. A semi rigid thoracoscope (LTF Type 160, Olympus) is used for the procedure. The other modality available is the rigid thoracoscope (which is not available in our centre). Firstly, patients were positioned in a lateral decubitus position with the affected side up. Bedside ultrasound was performed prior to the procedure to identify point for incision and feasibility for pleuroscopy. This point is located in the mid axillary line between the 4th and 7th intercostal space.

Following identification and preparation of the procedure site, local anaesthesia with $2 \%$ lignocaine was given. Afterward, a $1-2 \mathrm{~cm}$ incision was made followed by a blunt dissection. The trocar was then inserted once the pleural space was accessed. The pleuroscope was introduced into the pleural cavity via the trocar. The pleural cavity was inspected, and pleural biopsies were taken from the abnormal looking pleura (mass/ nodule). In cases where the pleura appeared normal, random biopsies may be taken.

After the procedure, chest tube was inserted and connected to an underwater seal drain. Correct placement of chest tube was confirmed by the swinging of pleural drainage and chest radiograph. The chest tube was then removed once the pleural fluid stop draining (or has drained less than $100 \mathrm{ml}$ over 24 hours) or once the lungs are fully expanded on subsequent chest radiograph.

\section{RESULT}

A total of 105 patients with effusion underwent pleuroscopy with a mean age of 60 (18) year-old (figure 1) and majority were male $(\mathrm{n}=60,57 \%)$. 97 patients had a pleural biopsy done while the remaining 8 patients only underwent a pleural fluid analysis (figure 2). Pleural biopsy and pleural fluid analysis were diagnostic in 94 patients $(90 \%)$ and pleural fluid analysis alone was diagnostic in 6 patients $(6 \%)$ (table 1). Among those who were diagnostic with pleural biopsy and pleural analysis, malignant etiologies were the commonest $(n=56$, 
$53 \%$ ) and with pleural fluid analysis alone (table

2) similar percentages were recorded for malignant and benign etiologies, $n=3(3 \%)$.

Adenocarcinoma was the commonest malignant finding in pleural biopsy and pleural analysis, and pleural fluid analysis; $n=47 \%$ $(53 \%)$ and $n=3(3 \%)$. Other malignant findings in pleural biopsy and pleural analysis were squamous cell carcinoma $4(4 \%)$, small cell carcinoma $3(3 \%)$, poorly differentiated carcinoma, $\mathrm{n}=1(1 \%)$ and yolk sac tumor, $\mathrm{n}=1$
(1\%). For non-malignant etiologies, granulomatous inflammation was the commonest finding, $\mathrm{n}=38(36 \%)$ followed by chronic inflammation, $\mathrm{n}=7(7 \%)$. Tuberculosis was diagnosed in 1 case based on pleural fluid alone. Malignant etiologies were more common in older age, 67(13) vs 50 (19) year-old (p 0.01) (Table 3).

With regards to safety profiles, only one patient developed re-expansion pulmonary edema and there were no procedure related mortalities. Furthermore, there were no complications such as subcutaneous emphysema, bleeding, or infection.

Figure 1. Patient age distribution

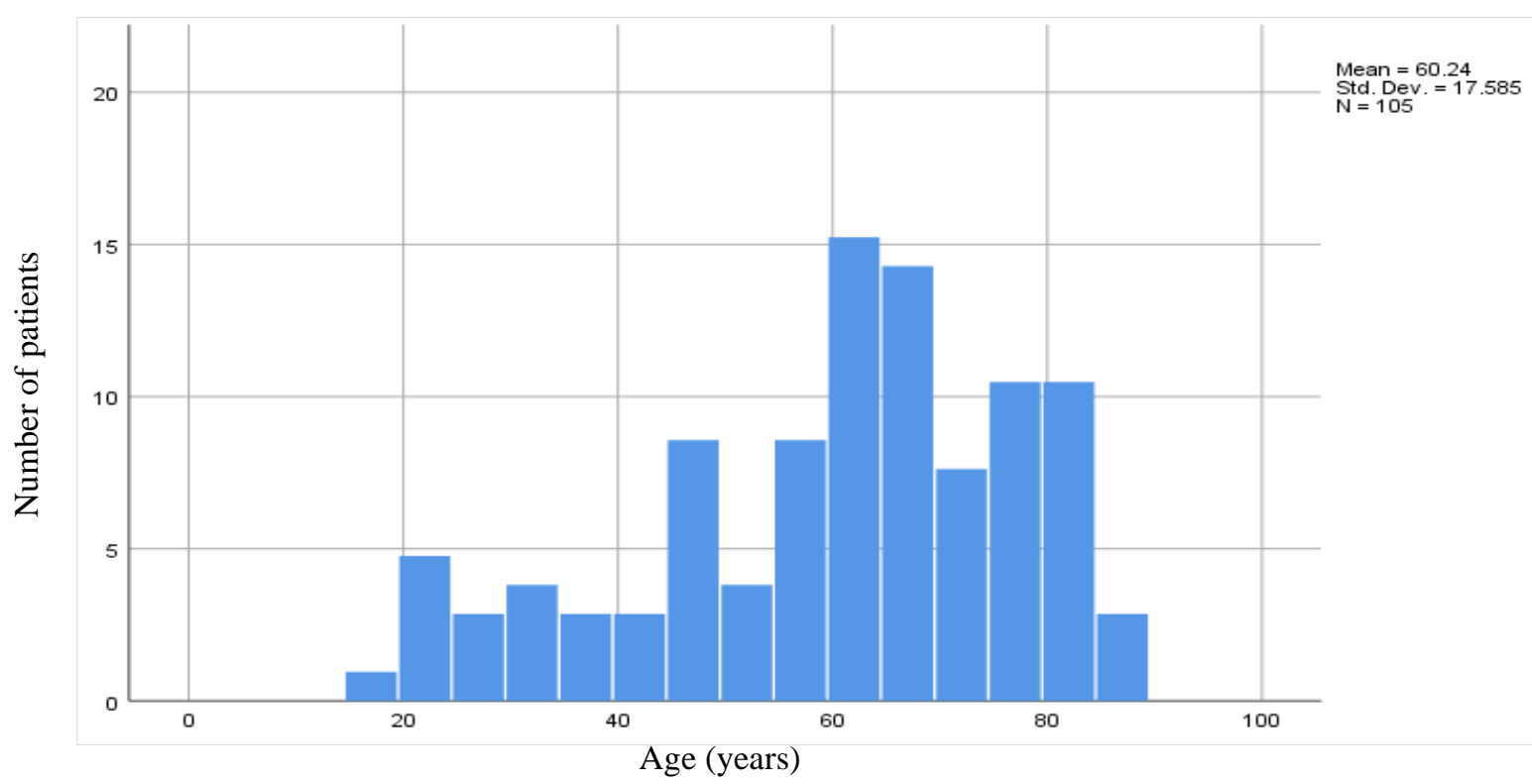

Figure 2. Flow chart of type of investigations underwent by patients

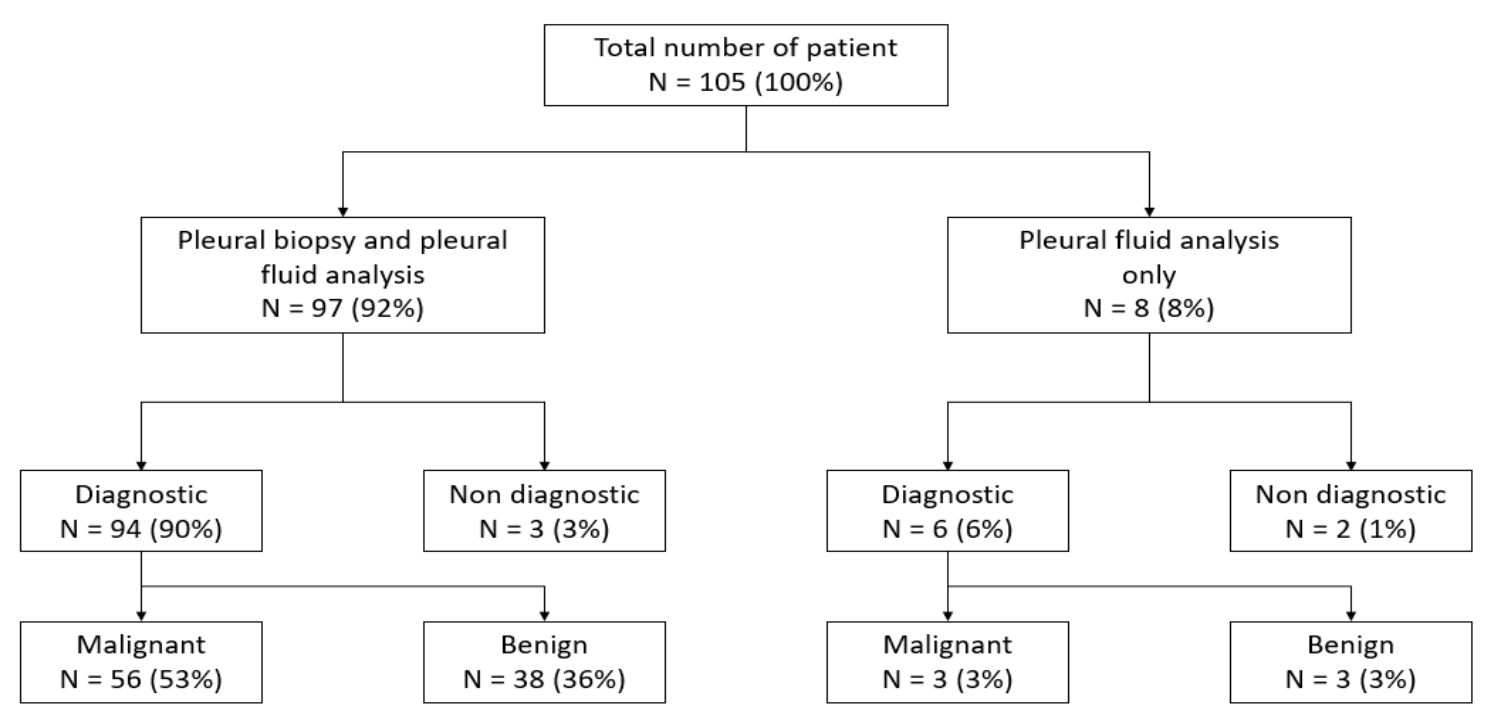


Table 1. Pleural biopsy and pleural fluid analysis $\mathrm{N}=97(92 \%)$

\begin{tabular}{|l|l|l|l|}
\hline & & $\mathbf{N}$ & $\mathbf{\%}$ \\
\hline Malignancy & & $\mathbf{5 6}$ & $\mathbf{5 3 \%}$ \\
\hline & Adenocarcinoma & 47 & $48.5 \%$ \\
\hline & Squamous cell carcinoma & 4 & $4.1 \%$ \\
\hline & Small cell carcinoma & 3 & $3.1 \%$ \\
\hline & Poorly differentiated carcinoma & 1 & $1.0 \%$ \\
\hline & Yolk sac tumour & 1 & $1.0 \%$ \\
\hline & & $\mathbf{3 8}$ & $\mathbf{3 6 \%}$ \\
\hline & Granulomatous inflammation & 18 & $18.6 \%$ \\
\hline & Chronic inflammation & 7 & $7.2 \%$ \\
\hline & Mesothelial cell & 7 & $7.2 \%$ \\
\hline & Fibrinous tissue & 3 & $3.1 \%$ \\
\hline & Atypical cell & 1 & $1.0 \%$ \\
\hline & Bony like tissue & 1 & $1.0 \%$ \\
\hline & Necrotic tissue & 1 & $1.0 \%$ \\
\hline & Inadequate sample & $\mathbf{3}$ & $\mathbf{3 . 1 \%}$ \\
\hline
\end{tabular}

Table 2. Pleural fluid analysis only $\mathrm{N}=8(8 \%)$

\begin{tabular}{|l|l|l|l|}
\hline & & $\mathbf{N}$ & $\mathbf{\%}$ \\
\hline Malignancy & Adenocarcinoma & $\mathbf{3}$ & $\mathbf{3 \%}$ \\
\hline Benign & & $\mathbf{3}$ & $\mathbf{3 \%}$ \\
\hline & Tuberculosis & 1 & $1 \%$ \\
\hline & Liver cirrhosis & 2 & $2 \%$ \\
\hline Inconclusive & & $\mathbf{2}$ & $\mathbf{2 \%}$ \\
\hline
\end{tabular}

Table 3. Comparison of age between malignant and benign etiologies. 5 patients were excluded due to non-diagnostic pleural fluid and biopsy result.

\begin{tabular}{|l|l|l|l|l|}
\hline Aetiologies & Number of patients & Mean age & Standard deviation & p-value \\
\hline Malignant & 66 & 67 & 13 & 0.01 \\
\hline Benign & 34 & 50 & 19 & \\
\hline
\end{tabular}




\section{DISCUSSION}

Exudative pleural effusion is a commonly encountered respiratory illness in day-to-day practice. Thoracocentesis is usually the first line of investigation however in up to $30 \%$ of cases it fails to provide the definitive diagnosis. Pleuroscopy provides a direct visualization of the pleural space and allows biopsy of the pleura to be taken under direct visualization. In cases of malignant pleural effusion, the sensitivity of pleuroscopy guided biopsy was reported between $81-95 \%$ (4-5). In this study, $97 \%$ of biopsy samples were diagnostic with only 3 samples obtained were non diagnostic This is similar to the other previous studies where adequate material were obtained in $93 \%-100 \%$ of the time $(1,2)$. Non diagnostic samples may be due to insufficient samples sent for analysis.

In the present study the biopsy results from pleuroscopy provide definitive diagnosis in $70 \%$ $(n=74)$ of the cases where diagnosis of malignancy $(\mathrm{N}=56)$ or tuberculosis $(\mathrm{N}=18)$ was made through histological analysis. This is slightly lower compared to the previous study (88-93\%) (1-2) (Gao et al and Sakuraba et al). This may be partly contributed by the fact that no pleural biopsy was taken in 8 of the cases. Unfortunately, we were unable to ascertain the reason to why pleural biopsy was not done in these 8 cases since it was a retrospective study.

Overall, malignancy is the most common cause for exudative pleural effusion comprising $56.2 \%(n=59)$ of cases. This percentage correlates and is slightly higher than other study where malignancy ranges between $45 \%-85 \%$ (2). This may be contributed by the fact that our patient pool belongs to the older category (mean age 60) compared to Gao Bao et al study group whereby the mean age is 46. Pleuroscopy also offers better tissue yield for additional mutation studies for targeted therapies especially in cases of advanced lung adenocarcinoma, whereby EGFR mutations are common in Asian population (6). Another reason for higher number of malignancy cases in our patient population can be attributed to selection bias whereby in some patients, pleuroscopy was not done if diagnosis of PTB has been made through sputum acid fast bacilli and hence this group of patients were excluded from our study.

Our data confirmed that the commonest diagnosis based on pleuroscopy is primary lung malignancy despite tuberculosis being rampant in our country. Interestingly, we specified the incidence of the different histologic subtypes. The adenocarcinoma of lung was confirmed to be the most common, comprising of more than $49.5 \%$ of the study sample.

Granulomatous inflammation was found in $19 \%(n=18)$ of the cases whereby all the patients were treated as pleural TB. None of them presented with recurrent pleural effusion. These patients were following up for a minimum duration of 6 months. Relying solely on Ziehl Nielsen staining of pleural fluid and pleural biopsy specimen is not helpful as the sensitivity was low, $0.0 \%$ and $3.8 \%$ respectively (7). However, pleural biopsy histopatholgy has a sensitivity of $53.8 \%$ and sensitivity of culture on both BACTEC 12B liquid medium and LJ medium was as high as $92.3 \%$ (7). Furthermore, molecular testing for tuberculosis using pleural fluid has a low sensitivity and high specificity (8). These facts support the use of pleuroscopy in the diagnosis of tuberculosis. However, the addition of MTB culture and sensitivity, MTB PCR and adenosine deaminase (ADA) result would be helpful to further confirm the diagnosis of tuberculosis. Based on current clinical practice, ADA test is only available in a private laboratory and patients have to pay for additional cost, thus making it less routinely sent during pleuroscopy.

We found that malignant pleural effusion is more commonly seen in the older age group; 67 vs 51 year-old ( $p$ 0.01) in comparison to other studies.

With regards to safety only one patient developed re-expansion pulmonary edema and there was no procedure related mortality. Apart from that, there were no complications such as 
subcutaneous emphysema, bleeding, or infection. Comparison to other studies. Thus, this study reaffirms that pleuroscopy is a safe procedure. Only 5 patients remained non-diagnostic post pleural fluid analysis and biopsy which require additional investigations.

\section{LIMITATIONS}

This study describes a single centre experience and retrospective data. We also could not ascertain reason for biopsy not taken and those loss to follow-up

\section{CONCLUSION}

To the best of our knowledge, this is the first study that illustrates the diagnostic yield directly on a cohort of patients and the safety of pleuroscopy in the diagnosis of pleural diseases in Northern Malaysia. We report data of more than 105 consecutive pleuroscopies over a 30 months duration. No patient selection was performed, and no follow-up time was considered except for pleural tuberculosis patients. Our cancer frequency $(56 \%)$ is similar to the one presented in existing literature. It suggests that the sensitivity in detecting malignancy of our procedure is as high as previously reported.

Pleuroscopy is a safe procedure and is a valuable modality in providing a diagnosis to the underlying cause of exudative pleural effusion. It can be easily done with minimal complications in a centre with experienced pulmonologists.

\section{REFERENCES:}

1) Sakuraba M, Masuda K, Hebisawa A, Sagara Y, Komatsu H. Thoracoscopic pleural biopsy for tuberculous pleurisy under local anesthesia. Annals of Thoracic and Cardiovascular Surgery. 2006 Jan $1 ; 12(4): 245$.

2) Gao BA, Zhou G, Guan L, Zhang LY, Xiang GM. Effectiveness and safety of diagnostic flexi-rigid thoracoscopy in differentiating exudative pleural effusion of unknown etiology: a retrospective study of 215 patients. Journal of thoracic disease. 2014 May;6(5):438.

3) Hirsch AP, Ruffie P, Nebut M, Bignon J, Chretien J. Pleural effusion: laboratory tests in 300 cases. Thorax. 1979 Feb 1;34(1):106-12.

4) Hucker J, Bhatnagar NK, Al-Jilaihawi AN, Forrester-Wood CP. Thoracoscopy in the diagnosis and management of recurrent pleural effusions. The Annals of thoracic surgery. 1991 Nov 1;52(5):11457.

5) Thomas M, Ibrahim WH, Raza T, Mushtaq K, Arshad A, Ahmed M, Taha S, Al Sarafandi S, Karim $\mathrm{H}$, Abdul-Sattar HA. Medical thoracoscopy for exudative pleural effusion: an eight-year experience from a country with a young population. BMC pulmonary medicine. 2017 Dec;17(1):1-5.

6) Liam CK, Leow HR, How SH, Pang YK, Chua KT, Lim BK, Lai NL, Kuan YC, Pailoor J, Rajadurai P. Epidermal growth factor receptor mutations in non-small cell lung cancers in a multiethnic malaysian patient population. Asian Pacific Journal of Cancer Prevention. 2014;15(1):321-6.

7) Hasaneen NA, Zaki ME, Shalaby HM, El-Morsi AS. Polymerase chain reaction of pleural biopsy is a rapid and sensitive method for the diagnosis of tuberculous pleural effusion. Chest. 2003 Dec 1;124(6):2105-11.

8) Rosso F, Michelon CT, Sperhacke RD, Verza M, Olival L, Conde MB, Guerra RL, Zaha A, Rossetti ML. Evaluation of real-time PCR of patient pleural effusion for diagnosis of tuberculosis. BMC research notes. $2011 \mathrm{Dec} ; 4(1): 1-6$. 